\title{
Experimental study on aging performance of elevator polyurethane buffer
}

\author{
Facai Ren*, Tao Jiang, Xiao Liang, Shaoyi Hou \\ Shanghai Institute of Special Equipment Inspection and Technical Research, Shanghai 200062, PR China
}

\begin{abstract}
In this paper, the hygrothermal aging experiments of polyurethane buffer materials for elevator were carried out for 5 days, 10 days, 15 days, 20 days, 25 days and 33 days. The quality change, hardness change, tensile strength and elongation at break of the test materials before and after the experiment were compared and analyzed. The results show that the aging process of polyurethane materials is accelerated by the humid and hot environment.
\end{abstract}

\section{Introduction}

Polyurethane elastomer as buffer material has been widely used in many fields. Polyurethane elastomers use microporous bubble structure to absorb energy to achieve buffering effect. Because polyurethane elastomer is a composite organic polymer material, polyurethane buffer has its characteristics of easy aging in the use process. Accelerated aging test of polyurethane buffer is very important to evaluate its performance in practical use. At present, many researchers use accelerated aging test to study the aging properties of different materials.

Gao et al. [1] studied the aging performance of modified polyurethane composite used in composite transmission tower. The results show that the combination of UV absorber and UV stabilizer can improve the aging properties of polyurethane composites. Grosjean et al. [2] studied the effects of temperature, irradiation, humidity and other single stress factors on the performance of solar materials by accelerated aging test. Pescheux et al. [3] analyzed the effect of four different anti pollution coatings on solar materials based on the results of several accelerated aging tests. Hayashi et al. [4] proposed an advanced accelerated aging test method for power devices. Park et al. [5] studied the aging properties of rubber materials and light rubber by accelerated aging test. The results show that the post yield stiffness and effective stiffness of small lead rubber bearings are higher than those of large lead rubber bearings. Wang et al. [6] proposed a new accelerated test method based on the thickness of epoxy resin material. Bauermann et al. [7] analyzed the conductive adhesive for solar cells using different accelerated aging tests. The results show that the hygrothermal degradation is related to the moisture adsorption. Xue et al. [8] studied the aging performance of glass fiber reinforced polymer connector in alkaline solution with different temperature and curing time by the accelerated aging test. The results show that the debonding between fiber and resin is more obvious with the increase of corrosion time and temperature.

In this paper, the accelerated aging test was used to study the polyurethane buffer material for elevator. The influence of different environmental parameters on the performance of elevator polyurethane buffer was analyzed.

\section{Material and Experimental}

The samples were taken from commercial elevator polyurethane buffer. The sample used for mass change test analysis is a cube with a side length of $100 \mathrm{~mm}$. The shape of the specimen used for tensile property testing is shown in Figure 1. The length of gauge section is $25 \mathrm{~mm}$, the width is $6 \mathrm{~mm}$ and the thickness is $2 \mathrm{~mm}$.

The condition of hygrothermal aging test is $65^{\circ} \mathrm{C}$ and $60 \%$ RH. The aging time was 5 days, 10 days, 15 days, 20 days, 25 days and 33 days. After the aging test, the properties of the samples were tested, including mass, Shore hardness, tensile strength and elongation at break. The test standard shall refer to GB/T 531.1-2008 < Rubber, vulcanized or thermoplastic-Determination of indentation hardness-Part 1: Duromerer method (Shore hardness) $>$ and GB/T 528-2009<Rubber, vulcanized or thermoplastic-Determination of tensile stress-strain properties $>$. The average value of the experimental results was calculated based on the results of three samples.

\footnotetext{
*Corresponding author e-mail: fcren@ssei.cn
} 


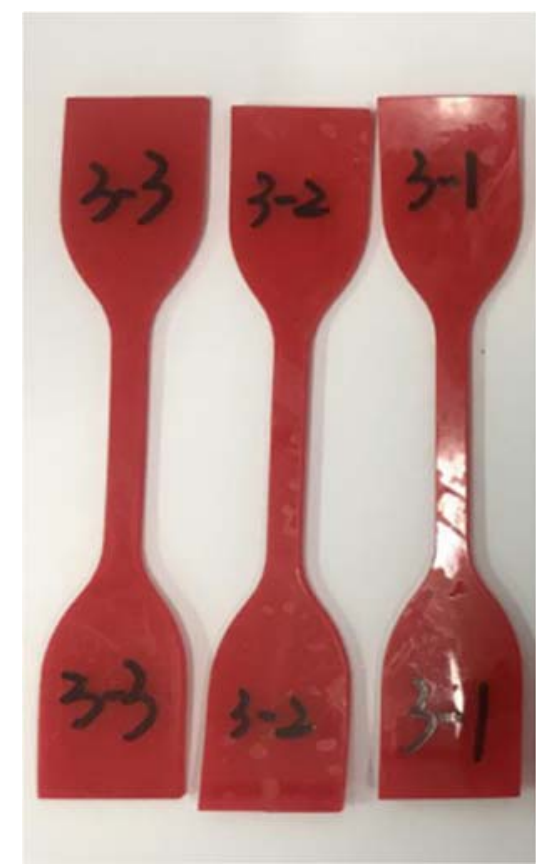

Fig 1. Geometry of the tensile test specimen.

\section{Results and Discussions}

\subsection{Tensile properties analysis}

The tensile strength of polyurethane materials after different time of hygrothermal aging test is tested, and the

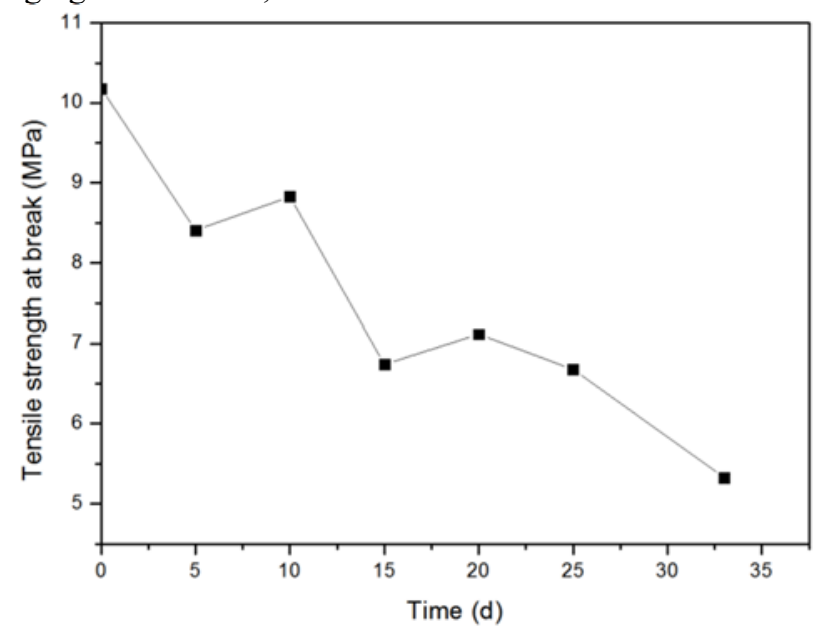

Fig 2. The tensile strength change of polyurethane after different hygrothermal aging time.

The elongation at break of polyurethane materials after different time of hygrothermal aging test is tested, and the results are shown in Fig. 3. The results showed that the elongation at break of polyurethane changed greatly with results are shown in Fig. 2. The results show that the tensile strength decreases obviously in the 0-5 days of hygrothermal aging test, and then increases with time, but the increase is not obvious. After that, the overall tensile strength showed a slow downward trend. time, and reached the highest value of $784 \%$ at 10 days of
hygrothermal aging test. After that, it showed a downward
trend. 


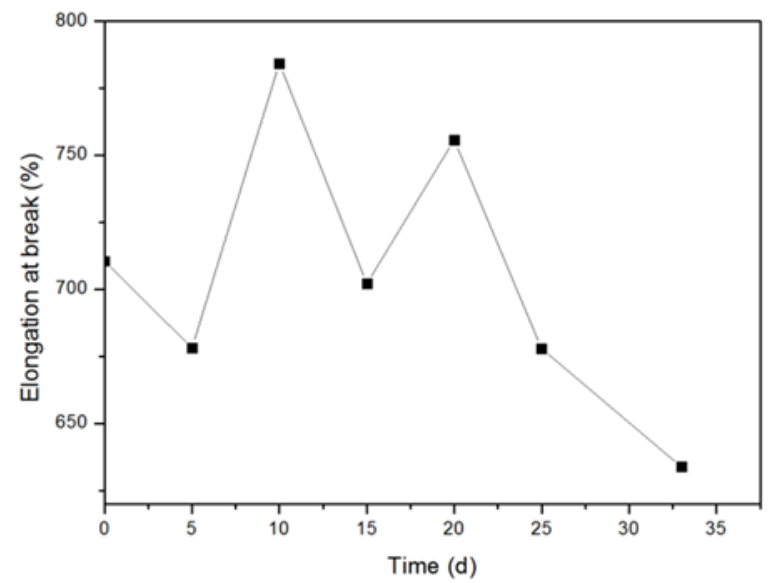

Fig 3. The elongation change of polyurethane after different hygrothermal aging time.

\subsection{Mass change analysis}

The samples were weighed after different time of hygrothermal aging test, and the mass change was shown in Fig. 4. The results show that the weight of polyurethane increases in different degrees after the hygrothermal aging test, which indicates that the polyurethane material is obviously affected by humidity.

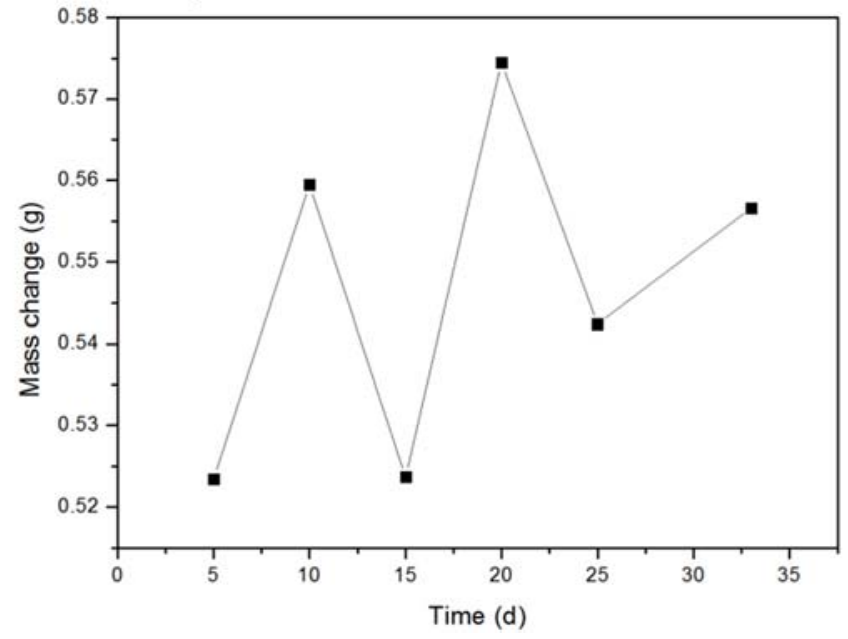

Fig 4. The mass change of polyurethane after different hygrothermal aging time.

\subsection{Hardness analysis}

The hardness of polyurethane after different time of hygrothermal aging test is tested, and the results are shown in Fig. 5. It can be seen that during the total test time of 33 days, the hardness change of polyurethane is small, and the shore hardness value slightly decreases.

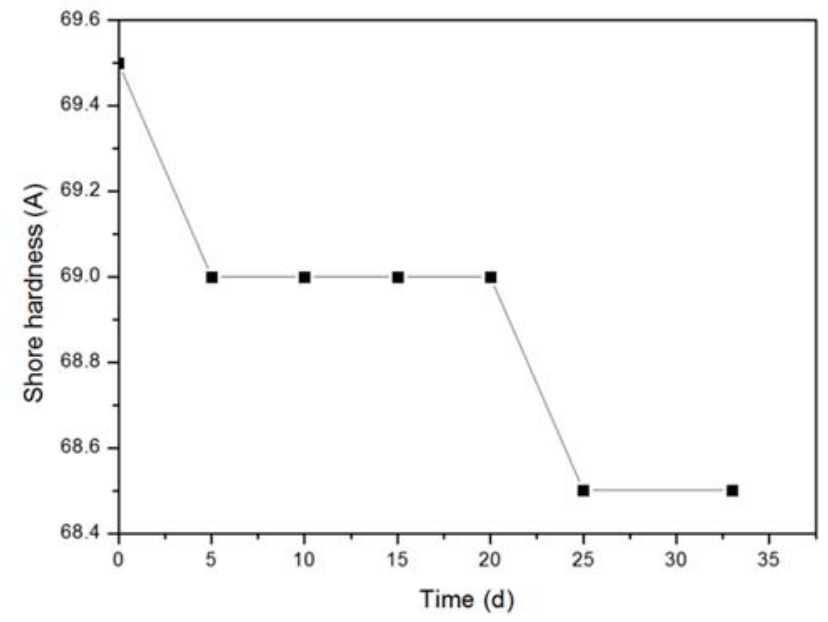

Fig 5. The hardness change of polyurethane after different hygrothermal aging time. 


\section{Conclusion}

The hygrothermal condition has certain influence on polyurethane materials. The results show that the aging rate of polyurethane materials is accelerated under humid and hot environment, and the tensile properties change obviously.

\section{Acknowledgments}

The authors are grateful for the support by Shanghai Bureau of Quality and Technical Supervision Research Project (No. 2019-26).

\section{References}

1. J.C. Gao, L.N. Wang, Y.Q. Fang, D.C. Huang, Y.Y. Ma, B. Song, L.Y. Wang, Aging performance investigation and improvement of modified polyurethane composites for composite overhead transmission line towers, Electr. Pow. Syst. Res. 191 (2021) 106880.

2. A. Grosjean, E.L. Baron, A.C. Pescheux, A. Disdier, Accelerated aging tests and characterizations of solar mirrors: Comparison of combinations of stress factors on degradation, Sol. Energ. Mat. Sol. C. 220 (2021) 110851 .

3. A.C. Pescheux, O. Raccurt, D. Bourdon, E.L. Baron, Accelerated aging tests and characterizations of innovated anti-soiling coatings for solar receiver glasses, Mater. Chem. Phys. 256 (2020) 123646.

4. S.I. Hayashi, K. Wada, Accelerated aging test for gate-oxide degradation in $\mathrm{SiC}$ MOSFETs for condition monitoring, Microelectron. Reliab. (2020) 113777.

5. J. Park, Y.S. Choun, M.K. Kim, D. Hahm, Revaluation of the aging property modification factor of lead rubber bearings based on accelerated aging tests and finite element analysis, Nucl. Eng. Des. 347 (2019) 59-66.

6. Y.L. Wang, X. Zhang, G.C. Cai, B.L. Wan, D. Waldmann, Y. Qu, A new thickness-based accelerated aging test methodology for resin materials: Theory and preliminary experimental study, Constr. Build. Mater. 186 (2018) 986-995.

7. L.P. Bauermann, E. Fokuhl, S. Stecklum, D. Philipp, T. Geipel, A. Kraft, U. Eitner, T. Fischer, D. Breitenbücher, Qualification of conductive adhesives for photovoltaic application - accelerated ageing tests, Energ. Procedia. 124 (2017) 554-559.

8. W.C. Xue, Y. Li, K. Fu, X. Hu, Y. Li, Accelerated ageing test and durability prediction model for mechanical properties of GFRP connectors in precast concrete sandwich panels, Constr. Build. Mater. 237 (2020) 117632. 